\title{
XLS (c9orf142) is a new component of mammalian DNA double-stranded break repair
}

\author{
A Craxton ${ }^{1}$, J Somers ${ }^{1}$, D Munnur ${ }^{1}, \mathrm{R}$ Jukes-Jones ${ }^{1}, \mathrm{~K}$ Cain $^{1}$ and M Malewicz ${ }^{* 1}$
}

Repair of double-stranded DNA breaks (DSBs) in mammalian cells primarily occurs by the non-homologous end-joining (NHEJ) pathway, which requires seven core proteins (Ku70/Ku86, DNA-PKcs (DNA-dependent protein kinase catalytic subunit), Artemis, XRCC4-like factor (XLF), XRCC4 and DNA ligase IV). Here we show using combined affinity purification and mass spectrometry that DNA-PKcs co-purifies with all known core NHEJ factors. Furthermore, we have identified a novel evolutionary conserved protein associated with DNA-PKcs-c9orf142. Computer-based modelling of c9orf142 predicted a structure very similar to XRCC4, hence we have named c9orf142-XLS (XRCC4-like small protein). Depletion of c9orf142/XLS in cells impaired DSB repair consistent with a defect in NHEJ. Furthermore, c9orf142/XLS interacted with other core NHEJ factors. These results demonstrate the existence of a new component of the NHEJ DNA repair pathway in mammalian cells.

Cell Death and Differentiation (2015) 22, 890-897; doi:10.1038/cdd.2015.22; published online 13 March 2015

Double-stranded DNA breaks (DSBs) are among the most cytotoxic DNA lesions for mammalian cells. ${ }^{1}$ Effective repair of DSBs is essential for cellular survival and for suppression of potential deleterious chromosomal rearrangements. ${ }^{2}$ Two main DNA repair pathways eliminate DSBs-homologous recombination (HR) or non-homologous end joining (NHEJ). HR utilises an undamaged copy of the chromosome as a template to direct repair, thus this restricts $H R$ to the $S$ and G2/M phases of the cell cycle, when such an extra chromosome copy is available. ${ }^{3}$ NHEJ performs the bulk of DSB repair in mammalian cells and in particular in during the G1 phase of the cell cycle, where the cells are completely dependent on NHEJ. NHEJ can be further subdivided into so-called classical NHEJ (c-NHEJ) and alternative NHEJ (alt-NHEJ). ${ }^{4}$ These DNA repair pathways utilise distinct protein components and also show different efficiencies of end ligation. In general, c-NHEJ is much more effective in end ligation than alt-NHEJ and can ligate most unrelated DNA ends directly or with minimal processing. In contrast alt-NHEJ requires short microhomologies between the DNA ends for ligation. ${ }^{5}$ C-NHEJ requires the following seven core proteins: Ku70/ Ku86 dimers, DNA-PKcs (DNA-dependent protein kinase catalytic subunit), Artemis nuclease, XRCC4-like factor (XLF) and the XRCC4/ligase IV complex. ${ }^{6,7}$ The DSB repair during $\mathrm{C}-\mathrm{NHEJ}$ is initiated by the Ku dimer that senses the presence of free double-stranded DNA ends in cells and rapidly binds such ends with high affinity. DNA-bound Ku then recruits DNAPKcs (DNA-PKcs/Ku70/Ku86 complex is termed DNA-PK holoenzyme), which has a protein kinase activity and is required for activation of the nuclease Artemis. ${ }^{8}$ Artemis, in turn, is responsible for DNA end processing in order to achieve DNA end structures suitable for ligation. The final step of C-NHEJ is the ligation of processed DNA ends by XRCC4/ ligase IV complex. This final step is stimulated by XLF protein that interacts with XRCC4 forming long filamentous structures at DSBs to facilitate DNA end joining. ${ }^{9,10}$ XRCC4 and XLF factors are distinct among NHEJ factors in that they share similar tertiary structure but show low primary sequence conservation. ${ }^{11}$ Since the identification of XLF in 2006, no new core factors have been discovered. ${ }^{11,12}$ Importantly, c-NHEJ is essential for proper development, as mutations in this pathway lead to immunodeficiency and defective neurogenesis in humans. ${ }^{7}$ It is therefore essential to fully decipher the identity of components for the c-NHEJ pathway and their regulation.

In this study, proteomic analysis of DNA-PKcs-containing protein complexes identified an abundant previously uncharacterised protein c9orf142, which we have named c9orf142XLS (XRCC4-like small protein). Structural modelling predicts XLS to be highly similar to XRCC4 and XLF, and depletion of XLS delays ionising radiation (IR)-induced DNA DSB repair. Moreover, XLS is associated with other core c-NHEJ factors. Our data strongly suggest that c9orf142/XLS represents a novel c-NHEJ component in mammalian cells.

\section{Results}

To identify and characterise novel DNA-PKcs-interacting proteins, we have generated a HEK293 cell line stably expressing N-terminal FLAG-tagged DNA-PKcs at physiological expression levels. Diluted nuclear extracts from these cells were subject to anti-FLAG immunoprecipitation (IP) followed by FLAG peptide elution and analysis by SDS-PAGE and silver staining (Figure 1a). FLAG-DNA-PKcs IPs contained several visible protein bands compared with control samples including 70 and $85 \mathrm{kDa}$ species, presumably corresponding to Ku70/Ku86 subunits of the DNA-PK holoenzyme. Immunoblotting of eluates from control and

${ }^{1}$ MRC Toxicology Unit, Hodgkin Building, Lancaster Road, Leicester LE1 9HN, UK

*Corresponding author: M Malewicz, MRC Toxicology Unit, Hodgkin Building, Lancaster Road, Leicester LE1 9HN, UK. Tel: +44 1162525579 ; Fax: +44 116 252 5616; E-mail: mm531@le.ac.uk

Abbreviations: NHEJ, non-homologous end joining; DNA-PKcs, DNA-dependent protein kinase catalytic subunit; XLF, XRCC4-like factor; XLS, XRCC4-like small protein; DSB, double-stranded DNA breaks; HR, homologous recombination; PMSF, phenylmethanesulphonyl fluoride; EtBr, ethidium bromide; IP, immunoprecipitation Received 24.1.15; accepted 09.2.15; Edited by G Melino; published online 13.3.15 
a

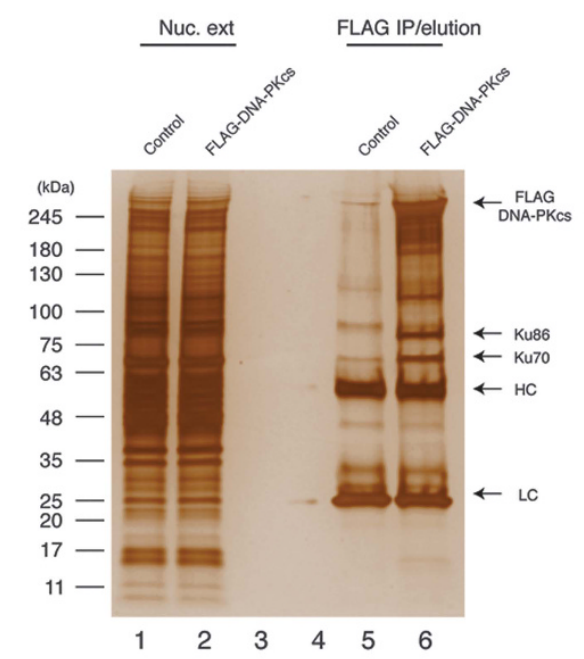

C

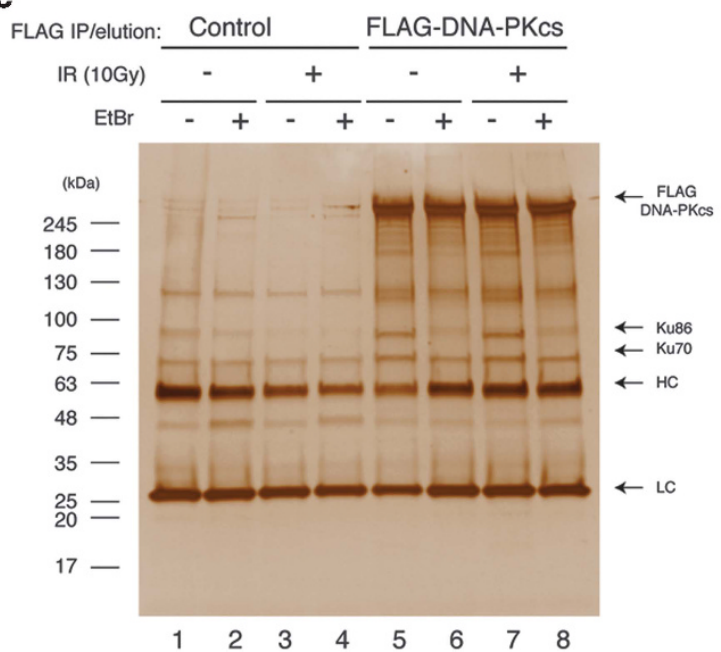

b

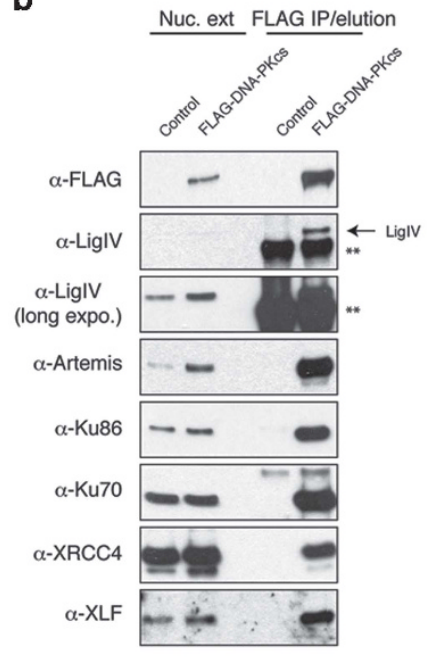

d

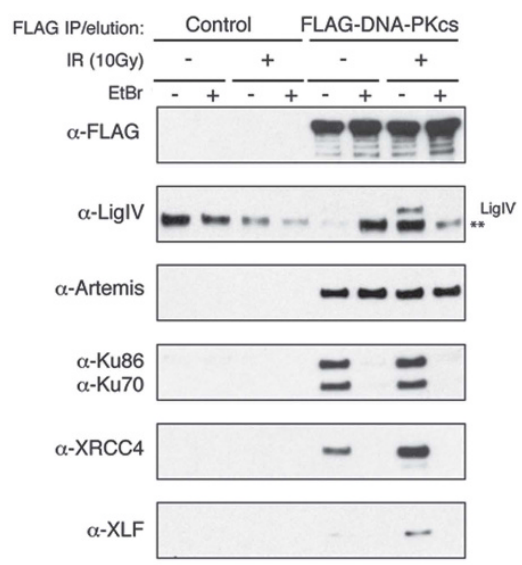

Figure 1 DNA-PKcs co-purifies with all known core NHEJ components. (a) Nuclear extracts (lanes 1 and 2) or eluates of purified anti-FLAG IPs from either HEK293 control (lane 5) or FLAG-DNA-PKcs-expressing cells (lane 6) were resolved by SDS-PAGE and visualised by silver staining. (b) As described in a, except that core NHEJ proteins were detected by immunoblotting. * Position of unspecific protein band. (c) Eluates of purified anti-FLAG IPs from either untreated (lanes 1 and 2) or irradiated (10 Gy, lanes 3 and 4) HEK293 control, or untreated (lanes 5 and 6) or irradiated (10 Gy, lanes 7 and 8) FLAG-DNA-PKcs-expressing cells were resolved by SDS-PAGE and visualised by silver staining. Nuclear extracts were incubated in the absence (lanes 1, 3, 5 and 7) or presence (lanes 2, 4, 6 or 8) of EtBr ( $0.2 \mathrm{mg} / \mathrm{ml}$ ) for 30 min before addition of anti-FLAG M2 beads. (d) As described in c, except that core NHEJ proteins were detected by immunoblotting. ${ }^{*}$ Position of unspecific protein band

FLAG-DNA-PKcs IPs identified all known core NHEJ components (Ku70/Ku86, Artemis, DNA Ligase IV, XRCC4 and XLF) specifically in FLAG-DNA-PKcs IPs (Figure 1b). Cellular DNA damage induced by IR is known to affect the stability of various DNA repair complexes by eliciting activation of stress signalling pathways, which catalyse many protein posttranslational modifications. ${ }^{3}$ Therefore, further experiments were performed under basal conditions or after exposing samples to IR. In order to assess the effect that contaminating DNA had on complex assembly, additional samples were treated with ethidium bromide (EtBr) before elution (Figure 1c). Although IR did not show obvious visible changes to the distribution of silver-stained bands, $\mathrm{EtBr}$ led to disappearance of several prominent protein bands including the 70- and 85$\mathrm{kDa}$ species, which we believe represent both Ku proteins (Figure 1c). Western blotting of core NHEJ factors revealed that Artemis directly interacted with DNA-PKcs as shown previously. ${ }^{13}$ All other core NHEJ proteins interacted with DNA-PKcs in a DNA-dependent fashion (Figure 1d). Interestingly, IR significantly increased the abundance of XLF and XRCC4/DNA ligase IV factors associated with DNA-PKcs, whereas the levels of Ku70/Ku86 and Artemis remained largely unchanged (Figure 1d). We reasoned that based upon our ability to detect all known core NHEJ factors in the FLAGDNA-PKcs IPs, it is possible that we may also detect previously uncharacterised core NHEJ components. To systematically analyse the protein composition of FLAGDNA-PKcs IPs, eluates were analysed by mass spectrometry to determine their proteomic composition. This proteomic analysis revealed the presence of large amounts of $\mathrm{Ku}$ proteins associated with DNA-PKcs (Figures 2a and d). The other most abundant proteins were PARP1, the FACT complex 
a

\begin{tabular}{|l|}
\hline \multicolumn{1}{|c|}{10 top } \\
Mass-spec hits list \\
\hline 1.
\end{tabular}

b

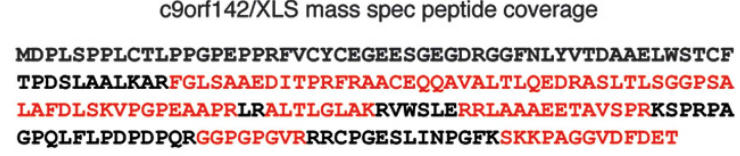

c

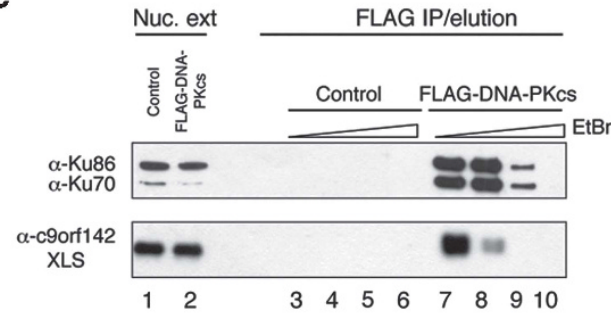

d
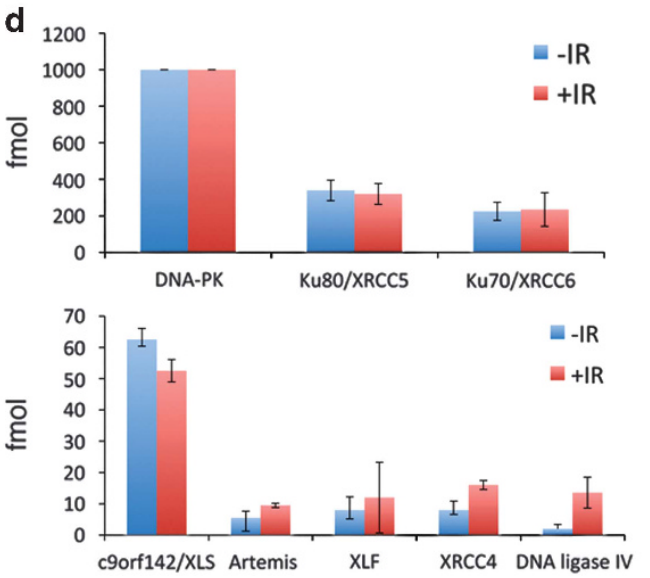

Figure 2 An uncharacterised protein c9orf142 associates with DNA-PKcs-containing NHEJ complexes. (a) Table showing 10 most abundant proteins identified in anti-FLAG IPs from FLAG-DNA-PKcs but not HEK293 control cells (average protein abundance from two independent experiments was used in creation of the ranking list). C9orf142 is shown in red (proteins are named based upon UniProt nomenclature. Cl142_HUMAN indicates c9orf142/XLS). (b) Sequence coverage for c9orf142/XLS. Amino acids, which were identified within peptides detected by LC-MS/MS, are marked in red. The total \% sequence coverage was $49 \%$. (c) Nuclear extracts (lanes 1 and 2 ) or eluates of isolated anti-FLAG IPs from nuclear extracts from either HEK293 control (lanes 3-6) or FLAG-DNA-PKcs expressing cells (lanes 7-10), which had been incubated with increasing concentrations of EtBr before addition of anti-FLAG beads were resolved by SDS-PAGE. Ku70, Ku86 and c9orf142 were detected by immunoblotting. (d) Identification of core NHEJ proteins from FLAG-DNA-PKcs IPs by LC-MS/MS mass spectrometry. Results shown in each column are the average quantities (fmol) of the indicated protein detected from duplicate injections from a total of four independent experiments

(SP16H and SSRP1) Werner protein (WRN) and protein phosphatase 6 regulatory subunit 3 (PP6R3) as previously shown (Figure 2a). ${ }^{14}$ Importantly, we also found substantial quantities of a previously uncharacterised protein c9orf142 consistently co-purifying with FLAG-DNA-PKcs (Figures 2a, b and d), which we have named XLS. Notably, compared with other core NHEJ factors, XLS was greater than fourfold, more abundant than XRCC4, XLF, DNA ligase IV and Artemis in the FLAG-DNA-PKcs IPs, and its levels were not increased in response to radiation in contrast to XRCC4, XLF and DNA ligase IV (Figures 1d and 2d). The presence of XLS in DNAPKcs IPs was verified by western blotting (Figure 2c). These results also demonstrated that XLS associated with DNAPKcs in a DNA-dependent manner, although interestingly the concentration of $\mathrm{EtBr}$ required for stripping XLS from the beads was significantly lower than the amount of EtBr needed for complete removal of Ku factors (Figure 2c).

We were intrigued by the high relative abundance of XLS in our DNA-PKcs IPs. Although primary sequence analysis of XLS did not reveal significant similarities to any known proteins, computer-based structural modelling using the Phyre2 algorithm ${ }^{15}$ revealed a structure of high similarity to XRCC4 (Figure 3a). It also showed structural resemblance to XLF, a known XRCC4 interactor, that forms filament-like structures with XRCC4. ${ }^{9,10}$ Although XLS is significantly smaller that XRCC4 (204 aa compared with 334 aa), it maintains similar overall predicted domain structure characterised by the presence of a $\mathrm{N}$-terminal head domain followed by a centrally located extended coiled coil and a C-terminal predicted bipartite nuclear localisation sequence (Figure $3 \mathrm{~b}$ ). The Phyre2 algorithm modelled amino acids 8-160 of XLS (ca. $75 \%$ of the fulllength XLS) with high confidence (ca. 95\%). However, the C-terminal portion of XLS (aa $161-204$ ) could not be modelled with high confidence and hence remained unstructured (Figure 3a). Modelling results obtained with the use of Phyre2 server were confirmed by subjecting XLS sequences to I-TASSER computer modelling platform (data not shown). The primary sequence of XLS is strongly phylogenetically conserved (Figure 3c) with surprisingly high-sequence conservation in the C-terminal region of XLS, which might suggest 
a

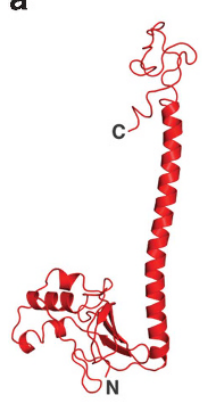

c9orf142/hXLS

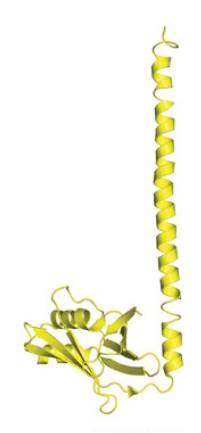

hXRCC4

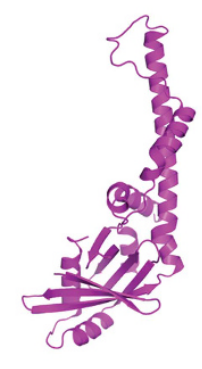

hXLF b

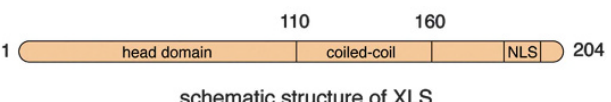

schematic structure of XLS

c

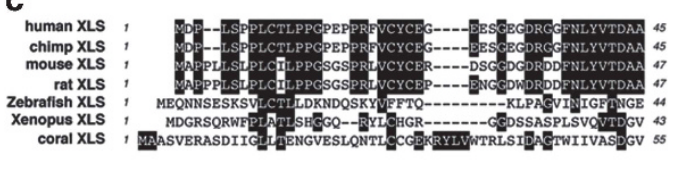

d
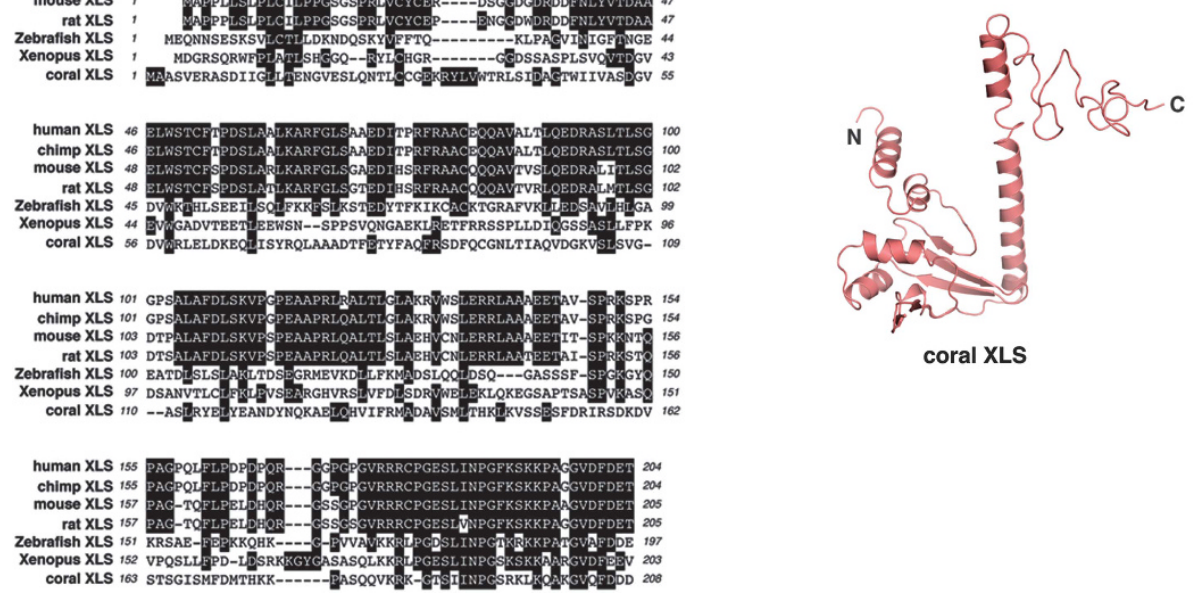

Figure 3 Computer modelling of $c 90$ orf142/XLS protein predicts a structure similar to XRCC4. (a) 3D structural model for full-length human c9orf142/XLS generated using the Phyre2 server, which predicted with $95 \%$ and $85 \%$ confidence overlapping structural features between amino acids 5-160 of human c9orf142/XLS with human XRCC4 and XLF (crystal structures), respectively. Although XRCC4 and XLF typically form dimers, the figure shows monomers for all proteins to highlight their structural similarities. ' $\mathrm{N}$ ' and 'C' indicate positions of the $\mathrm{N}$ - and $\mathrm{C}$ terminus of XLS, respectively. (b) Schematic figure showing the predicted structural and function domains for c9orf142/XLS. These are a $\mathrm{N}$-terminal head domain, a middle coiled-coil domain and a C-terminal nuclear localisation signal (NLS). (c) Primary sequence alignment of c9orf142/XLS from human, chimpanzee, mouse, rat, zebrafish, Xenopus and starlet sea anemone (coral) species. Identical amino acids are indicated as shaded black areas. (d) Predicted 3D structural model generated using Phrye2 server for full-length starlet sea anemone c9orf142/XLS, which shares $~ 20 \%$ sequence identity with human XLS (not shown)

essential functional and/or regulatory roles for this region of the protein. Evolutionary conservation of XLS structure can be further exemplified by its comparison with coral XLS orthologue. Although conservation of the protein sequence between the human and coral XLS is low (ca. 20\%; Figure 3c), the model for the coral XLS orthologue generated using the Phyre2 algorithm also predicted with high confidence similarity to XRCC4 (ca. $95 \%$ model confidence; Figure $3 d$ ).

The high relative abundance of XLS in DNA-PKcs IPs and its predicted striking structural similarity to XRCC4 led us to hypothesise that XLS may be involved in DSB repair. In order to assess the function of XLS, we first depleted XLS (and XRCC4 as a reference) using siRNA in two human cell types and observed efficient knockdown of XLS in both cell lines by qPCR and immunoblotting (Figure 4a). A typical phenotype of the NHEJ pathway deficiency is cellular radiosensitivity. ${ }^{7}$ Notably, knockdown of XLS in cells produced a radiosensitivity phenotype similar to depletion of XRCC4 measured by clonogenic survival assays (Figure $4 \mathrm{~b}$ ). To further establish similarity between XLS function and the activity of core NHEJ factors, we took advantage of the observation that XLFdeficient cells show substantially delayed resolution of histone $\mathrm{H} 2 \mathrm{AX}$ phosphorylation after radiation. ${ }^{11}$ We therefore assessed the kinetics of histone H2AX phosphorylation after IR in XLS-depleted cells. Knockdown of XLS delayed the disappearance of histone H2AX phosphorylation after IR consistent with defective DNA repair (Figure 4c). In order to confirm the DSB repair defect, we directly scored the repair kinetics of DSBs in XLS-depleted cells by visualisation of nuclear foci stained for phospho-Ser139-H2AX mark, a known DSB marker in eukaryotic cells. ${ }^{16}$ Depleting XLS led to a strong reduction in overall efficiency of DSB repair in cells (Figure 4d), closely resembling the previously reported phenotype of a human XLF mutant cell line. ${ }^{11}$ Collectively, these deficiencies in DSB repair suggest a defect in NHEJ.

To gain further evidence for XLS being a component of NHEJ, FLAG-tagged XLS was immunoprecipitated from nuclear extracts of HEK293S cells and IPs were probed for the presence of core NHEJ factors. Importantly, although XLS co-purified with DNA-PK subunits, XRCC4 and DNA ligase IV, 
a

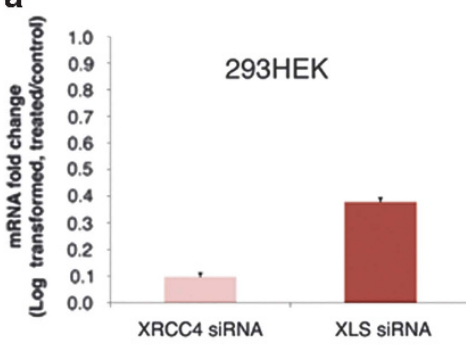

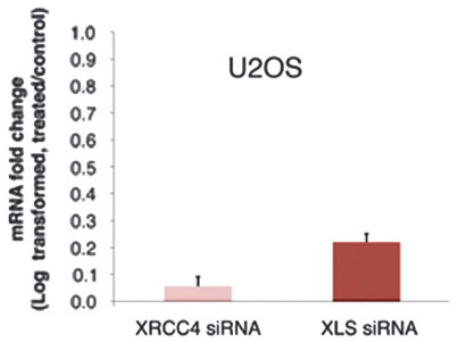

293HEK U2OS

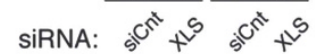
$\alpha-X L S$ $\alpha$-tubulin

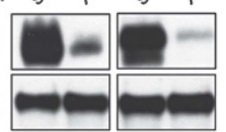

b

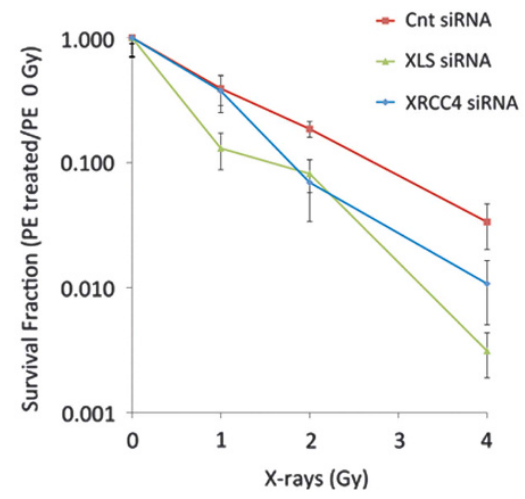

c

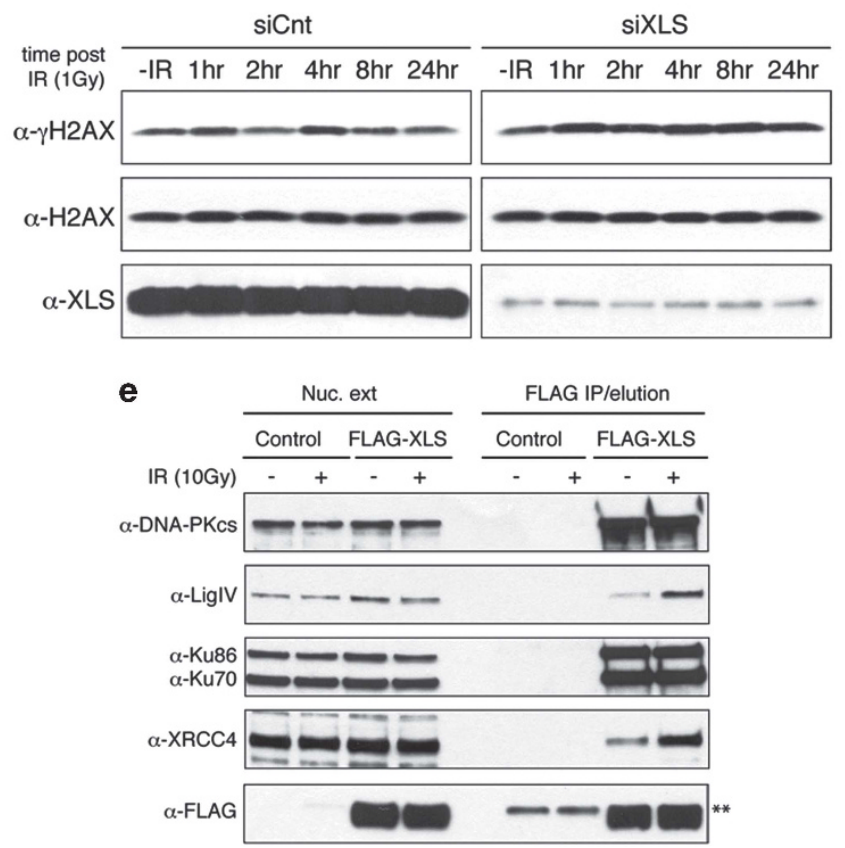

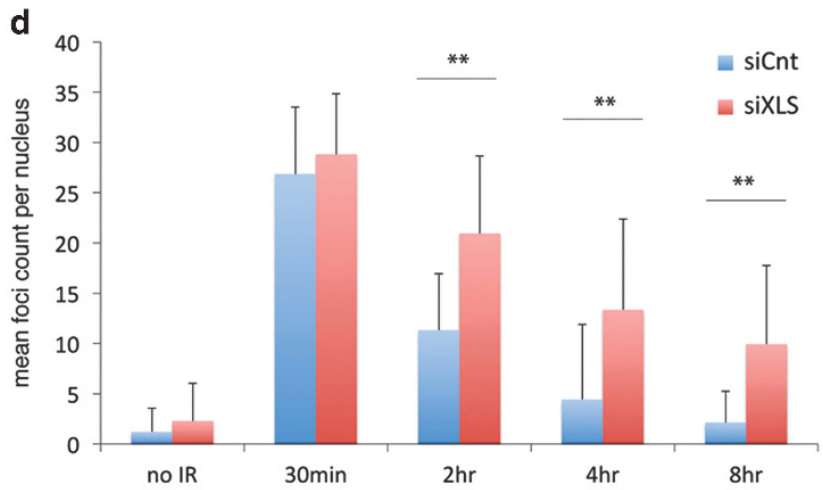

Figure 4 c9orf142/XLS protein interacts with most core NHEJ factors and its depletion leads to defective DNA DSB repair. (a) Upper panels: qPCR of XRCC4 and XLS mRNA expression after $72 \mathrm{~h}$ siRNA transfection in 293H and U2OS cells. Samples were normalised for GAPDH mRNA expression and expressed as mRNA fold change relative to control siRNA samples. Mean values and S.D. were plotted from three experimental repeats. Lower panels: western blot for XLS in control and XLS siRNA-transfected HEK293H and U2OS cells. (b) Clonogenic survival assay of $293 \mathrm{H}$ cells transfected with XLS, XRCC4 or control (Cnt) siRNA. Survival fraction was determined relative to 0 Gy control samples. Experiment was repeated three times. Representative experiment is shown. Mean values and S.D. were plotted. (c) XLS knockdown cells have elevated $\gamma \mathrm{H} 2 \mathrm{AX}$ levels following IR. U2OS cells were irradiated with X-rays (1 Gy) or left untreated. After the indicated times, histones were extracted from cells and immunoblotted for $\gamma \mathrm{H} 2 \mathrm{AX}$ or H2AX (loading control). XLS immunoblots were performed using the Triton X-100-soluble fractions. (d) XLS knockdown cells show slow $\gamma \mathrm{H} 2 \mathrm{AX}$ foci resolution following IR. U2OS cells were irradiated with X-rays (1 Gy) or left untreated (no IR). $\gamma \mathrm{H} 2 \mathrm{AX}$ foci were visualised by immunofluorescence. Experiment was repeated four times. Representative experiment is shown. Mean foci values per nucleus and S.D. were plotted. ${ }^{* *} P<0.005$. (e) XLS interacts with core c-NHEJ factors. HEK293S cells were transiently transfected for $20 \mathrm{~h}$ with either PCMX-LacZ (control) or PCMX-FLAG-XLS. Cells were untreated or irradiated with $10 \mathrm{~Gy}$ X-ray. After 30 min recovery at $37^{\circ} \mathrm{C}$, nuclear extracts were isolated. Following anti-FLAG IP and FLAG peptide elution, FLAG-XLS and associated proteins were eluted, resolved by SDS-PAGE and immunoblotted for the indicated core c-NHEJ factors. ${ }^{*}$ Position of IgG light chains cross-reacting with the secondary antibodies

treatment of cells with IR led to substantial increases in XRCC4/Ligase IV in FLAG-XLS IPs (Figure 4e).

\section{Discussion}

Historically most c-NHEJ factors have been discovered through complementation experiments, in which previously isolated mutant cell lines served as a critical starting point. ${ }^{17}$ A notable exception was the discovery of DNA ligase IV, which was found as a XRCC4-associated protein ${ }^{18}$ and the discovery of XLF by the Jackson group ${ }^{11}$ through a twohybrid approach. Here we have shown that immunoaffinity purification coupled with proteomics analysis by mass spectrometry can be successfully applied to identify new 
c-NHEJ proteins using a known NHEJ factor as bait (DNAPKcs in this instance). However, this method is not without caveats. For example, although we could detect Artemis nuclease in our FLAG-DNA-PKcs IPs, the overall yield of Artemis was comparatively low, thereby making it technically challenging to discover Artemis in an unbiased fashion among other proteins of low abundance present in the complex. This is in striking contrast to our discovery of c9orf142/XLS that robustly co-purified with DNA-PKcs and was consistently observed among the most abundant proteins detected (Figures 2a and d).

Here we have demonstrated that c9orf142/XLS is a new factor required for $\mathrm{DSB}$ repair in mammalian cells. Besides core proteins, the c-NHEJ DNA repair pathway uses additional socalled accessory factors (e.g., APLF (aprataxin and polynucleotide kinase/phosphatase-like factor) protein and NR4A orphan receptors $\left.{ }^{19,20}\right)$. These proteins typically serve to increase the efficiency of c-NHEJ without being absolutely essential. It appears possible that XLS represents a genuine core factor for c-NHEJ rather than an accessory molecule. This hypothesis is based on the observation that XLS is structurally similar to both XRCC4 and XLF (Figure 3a). Notably depletion of XLS produces a strong DSB repair defect and increased radiosensitivity, features uncommon for accessory NHEJ factors. ${ }^{19}$ However, more definitive proof for XLS being considered a core factor for c-NHEJ is nevertheless required. Other hallmarks of c-NHEJ deficiency include a shift in DSB repair to microhomology ${ }^{21}$ and a defect in somatic VDJ recombination in lymphocytes. ${ }^{22}$ With regard to VDJ recombination, it will be essential to assess the efficiency of this process in XLS knockout mice or potential human patients bearing inactivating mutations in the XLS-encoding gene. Precisely, how XLS functions within the c-NHEJ complex remains to be established. Understanding the pattern of XLS interactions among the c-NHEJ components will be important in deciphering its function in DSB repair. Accordingly, it is intriguing to note that, based on the relative abundance of $\mathrm{c}-\mathrm{NHEJ}$ factors in our DNA-PKcs IPs, it is interesting to speculate whether XLS may be directly bound to DNA-PKcs or via Ku, as only Ku factors are present in large enough quantities to mediate the bridging of XLS to DNA-PKcs. Given that interaction of XLS with DNAPKcs is DNA dependent (Figure 2c), it is more likely that XLS binds Ku subunits and/or DNA directly. Interestingly, FLAG-XLS co-purified with other c-NHEJ core factors, consistent with XLS being a core NHEJ component. Clearly, more experimental work needs to be done using XLS-deficient cells to draw a definitive conclusions regarding exact contribution of this protein to mammalian DSB repair.

While this manuscript was under preparation, another group reported the discovery of c9orf142 as a novel c-NHEJ factor. Although their functional data largely overlap with ours, their discovery of c9orf142 occurred through application of bioinformatics- and structure-guided approaches. ${ }^{23}$

\section{Materials and Methods}

Cells. HEK293 and U2OS cells were cultured in Dulbecco's-modified Eagle's medium, containing $4.5 \mathrm{~g} / \mathrm{D}$ D-glucose and GlutaMAX (Life Technologies, Carlsbad, CA, USA), and supplemented with $10 \%$ fetal bovine serum. HEK293 cell culture medium also contained pyruvate. A FLAG-DNA-PKcs-expressing stable cell line was generated by transfecting a mammalian expression vector encoding FLAG-
DNA-PKcs fusion protein (gift from Dr. David J. Chen, UTSouthwestern, Dallas, TX, USA) with pTKHyg plasmid into $293 \mathrm{H}$ cells (Life Technologies) and selecting individual clones with $200 \mu \mathrm{g} / \mathrm{ml}$ of hygromycin B (hygB). Individual hygB-resistant clones were screened for full-length FLAG-DNA-PKcs expression by western blotting. One clone expressing physiological levels of tagged-DNA-PKcs was selected for further experiments. Suspension-adapted HEK293 (HEK293S) cells were cultured in Freestyle 293 medium (Life Technologies) supplemented with hygB $(100 \mu \mathrm{g} / \mathrm{ml})$ between densities of $0.5-3 \times 10^{6}$ cells per $\mathrm{ml}$ in conical flasks on a shaking platform (160 r.p.m.) in a humidified $37^{\circ} \mathrm{C}$ incubator. HEK293S cells were transfected with pCMX-FLAG-XLS or PCMX-LacZ plasmid (gift from Dr. Thomas Perlmann, LICR, Stockholm, Sweden) using Freestyle MAX (Life Technologies) according to the manufacturer's instructions.

Plasmid constructs. Human full-length c9orf142/XLS cDNA was amplified with Q5 polymerase (NEB, Ipswich, MA, USA) by using oligo-dT-primed CDNA template from U2OS cells. The PCR product was subsequently subcloned into pCMX expression vector (gift from Dr. Thomas Perlmann, LICR). Sanger sequencing confirmed the insert sequence.

Antibodies. Mouse anti-FLAG (M2) and anti-tubulin (Sigma-Aldrich, St. Louis, MO, USA); mouse anti-Ku70, Ku86, DNA ligase IV, XLF and SPT16H (Santa Cruz Biotechnology, Dallas, TX, USA); rabbit anti-Artemis, PARP1 and $\gamma \mathrm{H} 2 \mathrm{AX}$ (Cell Signalling Technology, Danvers, MA, USA); c9orf142/XLS, DNA-PKcs and H2AX (Abcam, Cambridge, UK); and rabbit anti-XRCC4 was a generous gift from Dr. Dik Van Gent (Erasmus University, Rotterdam, Holland).

Immunoaffinity purification of FLAG-tagged DNA-PKcs and associated proteins from nuclear extracts. For each sample, 200$250 \times 10^{6}$ HEK293S cells stably expressing FLAG-DNA-PKcs or empty vector control were pelleted by centrifugation at $200 \times g$, resuspended in $20 \mathrm{ml}$ fresh media and placed in cell culture dishes and exposed to X-rays at 10 Gy (R320 Cabinet, $X$-strahl, Surrey, UK) or left untreated. Cells were subsequently incubated for $30 \mathrm{~min}$ at $37^{\circ} \mathrm{C}$. All subsequent procedures were performed on ice or at $4^{\circ} \mathrm{C}$. Cells were scraped into $15 \mathrm{ml}$ tubes, centrifuged at $200 \times \mathrm{g}$ for $5 \mathrm{~min}$ and washed twice in icecold PBS-MC (PBS, $1 \mathrm{mM} \mathrm{MgCl} 2$ and $1 \mathrm{mM} \mathrm{CaCl}$ ). Cells were gently resuspended in $3.6 \mathrm{ml}$ ice-cold hypotonic buffer (10 mM HEPES, pH 7.9, $10 \mathrm{mM} \mathrm{KCl}, 0.1 \mathrm{mM}$ EDTA and $0.1 \mathrm{mM}$ EGTA supplemented with complete Mini protease inhibitor mixture tablets (Roche Diagnostics, Burgess Hill, UK), $10 \mathrm{mM} \mathrm{NaF}, 1 \mathrm{mM} \mathrm{Na}_{3} \mathrm{VO}_{4}$, $10 \mu \mathrm{M}$ MG132, $1 \mathrm{mM}$ DTT and $1 \mathrm{mM}$ phenylmethanesulphonyl fluoride (PMSF)). After incubation for $15 \mathrm{~min}$, cells were vortexed for $10 \mathrm{~s}$, and immediately centrifuged at $2300 \times g$ for $5 \mathrm{~min}$ at $4^{\circ} \mathrm{C}$. Nuclei were washed with $1 \mathrm{ml}$ hypotonic buffer and re-centrifuged as described above. Pellets were overlayed with $4 \mathrm{ml}$ high salt buffer (20 mM HEPES, pH 7.9, $420 \mathrm{mM} \mathrm{NaCl}, 1.5 \mathrm{mM} \mathrm{MgCl}_{2}$ and $20 \%$ glycerol supplemented with complete Mini protease inhibitor mixture tablets (Roche Diagnostics), $10 \mathrm{mM} \mathrm{NaF}, 1 \mathrm{mM} \mathrm{Na}_{3} \mathrm{VO}_{4}, 10 \mu \mathrm{M} \mathrm{MG} 132,1 \mathrm{mM}$ DTT and $1 \mathrm{mM}$ PMSF) for 15 min with occasional mixing to resuspend crude nuclei. Following centrifugation at $15000 \times g$ for $30 \mathrm{~min}$, high salt nuclear extracts were diluted with 2 volumes of $20 \mathrm{mM}$ HEPES, pH 7.9, $20 \%$ glycerol and supplemented with $0.5 \%$ Igepal CA630, and subsequently incubated for an additional $30 \mathrm{~min}$ and recentrifuged at $15000 \times g$ for $30 \mathrm{~min}$. Protein concentrations were quantified using Bradford Reagent (Bio-Rad, Hercules, CA, USA) and equal quantities were incubated overnight by end-to-end mixing at $4^{\circ} \mathrm{C}$ with $25 \mu$ l of low pH glycinewashed packed anti-FLAG M2 agarose beads according to the manufacturer's instructions (Sigma-Aldrich). Beads were washed five times with $20 \mathrm{mM} \mathrm{HEPES,} \mathrm{pH}$ 7.9, $140 \mathrm{mM} \mathrm{NaCl}, 0.5 \mathrm{mM} \mathrm{MgCl}_{2}, 20 \%$ glycerol, $10 \mathrm{mM} \mathrm{NaF}, 1 \mathrm{mM} \mathrm{Na}_{3} \mathrm{VO}_{4}, 10 \mu \mathrm{M}$ MG132, $1 \mathrm{mM}$ DTT and $1 \mathrm{mM}$ PMSF, and proteins were eluted with $50 \mu \mathrm{l}$ 3X FLAG peptide $(0.2 \mathrm{mg} / \mathrm{ml})$. In some experiments, EtBr was added to either incrementally reduce DNA binding $(5-200 \mu \mathrm{g} / \mathrm{ml})$ or completely suppress DNA-dependent binding $(200 \mu \mathrm{g} / \mathrm{ml})$. Eluates were resolved by SDS-PAGE and gels were visualised with either silver stain (Pierce, Rockford, IL, USA) or for mass spectrometry stained with colloidal Coomassie (National Diagnostics, Hessle, UK).

Identification of DNA-PKcs-interacting proteins using LC-MS/MS. After destaining with deionised $\mathrm{H}_{2} \mathrm{O}$ according to the manufacturer's instructions, gels were serially sectioned, digested with trypsin and peptides were extracted as described previously. ${ }^{24,25}$ Dried tryptic peptides were resuspended in $5 \%$ formic acid and $10 \%$ acetonitrile $(9: 1)$, spiked with $20 \mathrm{fmol} / \mu \mathrm{l}$ MassPREP standards (Waters Corporation, Manchester, UK), using yeast ADH1 (accession no. P00330) and bovine serum albumin (accession no. P02769), Nanoscale UPLC separation of 
tryptic peptides was carried out on a nanoAcquity UPLC system (Waters Corporation) equipped with a $25 \mathrm{~cm} \times 75 \mu \mathrm{m}$ I.D., $1.7 \mu \mathrm{m}, \mathrm{BEH} 130 \mathrm{C} 18$ analytical reverse phase column. Samples (2-4 $\mu$ linjections) were separated using 90-min, $3-40 \%$ acetonitrile gradients at $0.3 \mu \mathrm{l} / \mathrm{min}$. Mass spectrometric analysis of eluted peptides, using a Waters Synapt G2-S HDMS mass spectrometer (Waters Ltd, Elstree, UK), equipped with T-Wave-IMS and carried out in data-independent acquisition and ion mobility modes $\left(\mathrm{HDMS}^{\mathrm{E}}\right)$, with a travelling wave velocity of $650 \mathrm{~m} / \mathrm{s}$. Peptide fragmentation was performed, by stepping between $4 \mathrm{eV}$ (low energy) and $20-50 \mathrm{eV}$ (collision-induced dissociation) voltages. Low-energy and CID data were acquired with a 1-s cycle scan time and $50-2000 \mathrm{~m} / \mathrm{z}$ mass range. LC-MS data were processed and searched using Waters ProteinLynx Global SERVER version 3.0 (PLGS, Waters) and identified, using the UniProt Human reviewed database (UniProtKB release 2014_11, 20265 entries). Raw data files were analysed using PLGS version 3 and ISOQuant. ${ }^{26}$ These data were used for 'top 3 ' absolute quantification of proteins. ${ }^{27}$ For database searching in PLGS, peptide mass tolerance and fragment mass tolerance were set to auto, with one missed cleavage and variable modification for methionine oxidation. A falsediscovery rate (FDR) of $1 \%$ was used for PLGS and for ISOQuant analysis an FDR of $0.1 \%$ was used with only TOP 3 peptide hits used for quantification. Data were also analysed using Scaffold version 3.3.1 software (Proteome Software Inc., Portland, OR, USA) as previously described. ${ }^{25}$

Computer modelling, visualisation and multiple sequence alignment of proteins. Computer modelling was performed by submitting XLS protein sequences to Phyre2 server..$^{15}$ For both human (accession no. NP_899064) and coral (accession no. XP_001641097, starlet sea anemone) XLS models with a high degree of probability of correctness were achieved (ca. 95\%). Results obtained with the use of Phre2 server were confirmed by subjecting XLS sequences to I-TASSER computer modelling platform (http://zhanglab.ccmb.med.umich.edu). Figures $3 a$ and $d$ were generated using PyMOL Molecular Graphic System, version 1.6.0.0 (Schrödinger, LLC, Camberley, UK). The Phyre2-generated models of human and coral XLS were compared with the crystal structures of XRCC4; PDB accession codes $1 F U 1^{28}$ and XLF; PDB accession code $2 R_{9}$. $^{29}$ XLS protein sequences were aligned using ClustalW algorithm from within MacVector sequence analysis software (MacVector Inc., Cary, NC, USA). Human XLS (accession no. NP_899064), chimp XLS (accession no. XP_009456033), mouse XLS (accession no. NP_705785), rat XLS (accession no. XP_006233650), zebrafish XLS (accession no. NP_001124069) and Xenopus XLS (accession no. XP_004917607) were used for alignment.

siRNA-mediated knockdown experiments. Cells were transfected with $20 \mathrm{nM}$ ON-TARGETplus siRNA SMARTpools (GE Healthcare, Little Chalfont, UK) using Lipofectamine RNAiMAX (Life Technologies) according to the manufacturer's protocol.

Clonogenic survival assays. Seventy-two hours following siRNA transfection, cells were replated in triplicate at low density in six-well plates. Once attached, cells were exposed to X-rays and grown for 11 days to form colonies. Colonies were fixed in $75 \%$ methanol : $25 \%$ acetic acid, before staining with $0.05 \%(\mathrm{w} / \mathrm{v})$ crystal violet. Plates were scanned using an Odyssey CLX imaging system (LI-COR Biosciences, Lincoln, NE, USA) and plate intensities (as a measure of colony formation) were determined with the ColonyArea Plugin ${ }^{30}$ and Image-J software (National Institutes of Health, Bethesda, MD, USA). The survival fraction was determined from the plating efficiency of the treatment relative to the plating efficiency of the 0 -Gy controls.

DSB repair foci counting. Cells were transfected with siRNA reagents and then $48 \mathrm{~h}$ later plated at the density of $35 \times 10^{3}$ cells per well on Lab-Tek II 8-well chamber slides (Nunc, ThermoFisherScientific, Rugby, UK) in $500 \mu$ l of complete medium. After overnight incubation, cells were exposed to 1 Gy of X-rays (R320 Cabinet, $\mathrm{X}$-strahl) and further cultured at $37^{\circ} \mathrm{C}$. At indicated time points cells were fixed with $4 \%$ paraformaldehyde directly on slides for $10 \mathrm{~min}$ at room temperature. Cells were subsequently washed with PBS and incubated at $4{ }^{\circ} \mathrm{C}$ in blocking buffer ( $5 \%$ normal goat serum, $0.3 \%$ Triton X-100 in PBS) for at least 30 min. Cells were further incubated with primary antibodies diluted in blocking buffer at $4^{\circ} \mathrm{C}$ (rabbit anti-phospho-Ser139-H2AX Ab; Cell Signalling Technology). After overnight incubation, cells were washed in PBS at room temperature and then secondary antibodies (goat anti-rabbit Cy3-coupled Abs; Jackson ImmunoResearch, West Grove, PA, USA) were applied at room temperature in blocking buffer containing DAPI (Sigma-Aldrich) for $1 \mathrm{~h}$. Finally, cells were washed in PBS and mounted in Vectashield-mounting medium (Vector Labs, Peterborough, UK). Images of nuclei were acquired on Zeiss LSM510 (Carl Zeiss Ltd., Cambridge, UK) and then individual DSB foci were counted per nucleus. To quantify DSB repair defect, 50 nuclei were scored per data point and mean values \pm S.D. were plotted. Data were statistically analysed using two-sided paired $T$-test.

qPCR. Seventy-two hours post-siRNA transfection, RNA was extracted using QIAshredder columns and the RNeasy mini kits (Qiagen, Venlo, Holland) according to manufacturer's instructions. cDNA was reverse transcribed using anchored oligo $\mathrm{dT}^{20}$ primer and Superscript II (Life Technologies) following the manufacturer's instructions. qPCR was performed using SYBR green (Applied Biosystems, Paisley, UK) and measured on a QuantStudio 6 Flex Real-Time machine (Life Technologies). Samples were normalised to GAPDH mRNA and the fold change between the gene-specific siRNA and the control siRNA samples was determined. ${ }^{20}$ qPCR primers: XLS forward 5'-GAGAGTCGCTCATCAACCCC-3', XLS reverse 5'-AAAGACTGCCTCTCCCCTCA-3'; XRCC4 and GAPDH primer sequences were obtained from literature. ${ }^{31,32}$

Extraction of histones for Immunoblotting. Cells were resuspended in Triton extraction buffer (TEB; PBS, 0.5\%(v/v) Triton X-100, 2 mM PMSF, $10 \mathrm{mM} \mathrm{NaF}$, $1 \mathrm{mM} \mathrm{Na}_{3} \mathrm{VO}_{4}$ and $0.02 \% \mathrm{NaN}_{3}$, and lysed on ice for 10 min with intermittent mixing. After centrifugation at $6500 \times g$ for $10 \mathrm{~min}$ at $4^{\circ} \mathrm{C}$, nuclear pellets were washed with half the volume of TEB and re-centrifuged. Pellets were resuspended in $0.2 \mathrm{~N} \mathrm{HCl}$ and incubated overnight at $4^{\circ} \mathrm{C}$. Following centrifugation at $6500 \times \mathrm{g}$ for $10 \mathrm{~min}$ at $4^{\circ} \mathrm{C}$, the protein concentration of supernatants was quantified by Bradford assay (Bio-Rad).

\section{Conflict of Interest}

The authors declare no conflict of interest.

Acknowledgements. We would like to thank Dr David J. Chen, Dr Benjamin Chen, Dr Dik Van Gent and Dr Thomas Perlmann for generously sharing reagents. Medical Research Council, UK, funds Michal Malewicz work.

1. Bekker-Jensen $S$, Mailand N. Assembly and function of DNA double-strand break repair foci in mammalian cells. DNA Repair (Amst) 2010; 9: 1219-1228.

2. Grundy GJ, Moulding HA, Caldecott KW, Rulten SL. One ring to bring them all-the role of Ku in mammalian non-homologous end joining. DNA Repair (Amst) 2014; 17: 30-38.

3. Ciccia A, Elledge SJ. The DNA damage response: making it safe to play with knives. Mol Cell 2010; 40: 179-204.

4. Pannunzio NR, Li S, Watanabe G, Lieber MR. Non-homologous end joining often uses microhomology: implications for alternative end joining. DNA Repair (Amst) 2014; 17: 74-80.

5. Frit P, Barboule N, Yuan Y, Gomez D, Calsou P. Alternative end-joining pathway(s): bricolage at DNA breaks. DNA Repair (Amst) 2014; 17: 81-97.

6. Waters CA, Strande NT, Wyatt DW, Pryor JM, Ramsden DA. Nonhomologous end joining: a good solution for bad ends. DNA Repair (Amst) 2014; 17: 39-51.

7. Woodbine L, Gennery AR, Jeggo PA. The clinical impact of deficiency in DNA non-homologous end-joining. DNA Repair (Amst) 2014; 16: 84-96.

8. Davis AJ, Chen BP, Chen DJ. DNA-PK: a dynamic enzyme in a versatile DSB repair pathway. DNA Repair (Amst) 2014; 17: 21-29.

9. Ochi $T, W u Q$, Blundell TL. The spatial organization of non-homologous end joining: from bridging to end joining. DNA Repair (Amst) 2014; 17: 98-109.

10. Hammel M, Yu Y, Fang S, Lees-Miller SP, Tainer JA. XLF regulates filament architecture of the XRCC4.ligase IV complex. Structure 2010; 18: 1431-1442.

11. Ahnesorg $P$, Smith $P$, Jackson SP. XLF interacts with the XRCC4-DNA ligase IV complex to promote DNA nonhomologous end-joining. Cell 2006; 124: 301-313.

12. Buck D, Malivert L, de Chasseval R, Barraud A, Fondaneche MC, Sanal O et al. Cernunnos, a novel nonhomologous end-joining factor, is mutated in human immunodeficiency with microcephaly. Cell 2006; 124: 287-299.

13. Ma Y, Pannicke U, Schwarz K, Lieber MR. Hairpin opening and overhang processing by an Artemis/DNA-dependent protein kinase complex in nonhomologous end joining and V(D)J recombination. Cell 2002; 108: 781-794.

14. Douglas P, Zhong J, Ye R, Moorhead GB, Xu X, Lees-Miller SP. Protein phosphatase 6 interacts with the DNA-dependent protein kinase catalytic subunit and dephosphorylates gamma-H2AX. Mol Cell Biol 2010; 30: 1368-1381.

15. Kelley LA, Sternberg MJ. Protein structure prediction on the Web: a case study using the Phyre server. Nat Protoc 2009; 4: 363-371.

16. Bekker-Jensen S, Lukas C, Kitagawa R, Melander F, Kastan MB, Bartek J et al. Spatial organization of the mammalian genome surveillance machinery in response to DNA strand breaks. J Cell Biol 2006; 173: 195-206. 
17. Jackson SP. Colworth Medal lecture. Detection, repair and signalling of DNA doublestrand breaks. Biochem Soc Trans 1999; 27: 1-13

18. Grawunder U, Wilm M, Wu X, Kulesza P, Wilson TE, Mann M et al. Activity of DNA ligase IV stimulated by complex formation with XRCC4 protein in mammalian cells. Nature 1997; 388 : 492-495.

19. Rulten SL, Fisher AE, Robert I, Zuma MC, Rouleau M, Ju L et al. PARP-3 and APLF function together to accelerate nonhomologous end-joining. Mol Cell 2011; 41: 33-45.

20. Malewicz M, Kadkhodaei B, Kee N, Volakakis N, Hellman U, Viktorsson K et al. Essential role for DNA-PK-mediated phosphorylation of NR4A nuclear orphan receptors in DNA double-strand break repair. Genes Dev 2011; 25: 2031-2040.

21. Fattah FJ, Kweon J, Wang $\mathrm{Y}$, Lee EH, Kan $\mathrm{Y}$, Lichter $\mathrm{N}$ et al. A role for XLF in DNA repair and recombination in human somatic cells. DNA Repair (Amst) 2014; 15 : $39-53$

22. Dudley $\mathrm{DD}$, Chaudhuri J, Bassing $\mathrm{CH}$, Alt FW. Mechanism and control of $\mathrm{V}(\mathrm{D}) J$ recombination versus class switch recombination: similarities and differences. Adv Immunol 2005; 86: 43-112.

23. Ochi T, Blackford AN, Coates J, Jhujh S, Mehmood S, Tamura N et al. DNA repair. PAXX, a paralog of XRCC4 and XLF, interacts with Ku to promote DNA double-strand break repair. Science 2015; 347: 185-188.

24. Boyd RS, Jukes-Jones R, Walewska R, Brown D, Dyer MJ, Cain K. Protein profiling of plasma membranes defines aberrant signaling pathways in mantle cell lymphoma. Mol Cell Proteom 2009; 8: 1501-1515.

25. Dickens LS, Boyd RS, Jukes-Jones R, Hughes MA, Robinson GL, Fairall $L$ et al. A death effector domain chain DISC model reveals a crucial role for caspase-8 chain assembly in mediating apoptotic cell death. Mol Cell 2012; 47: 291-305.

26. Distler U, Kuharev J, Navarro P, Levin Y, Schild H, Tenzer S. Drift time-specific collision energies enable deep-coverage data-independent acquisition proteomics. Nat Methods 2014; 11: 167-170.
27. Silva JC, Denny R, Dorschel C, Gorenstein MV, Li GZ, Richardson K et al. Simultaneous qualitative and quantitative analysis of the Escherichia coli proteome: a sweet tale. Mol Cell Proteom 2006; 5: 589-607.

28. Junop MS, Modesti M, Guarne A, Ghirlando R, Gellert M, Yang W. Crystal structure of the Xrcc4 DNA repair protein and implications for end joining. EMBO J 2000; 19: 5962-5970.

29. Andres SN, Modesti M, Tsai CJ, Chu G, Junop MS. Crystal structure of human XLF: a twist in nonhomologous DNA end-joining. Mol Cell 2007; 28: 1093-1101.

30. Guzman C, Bagga M, Kaur A, Westermarck J, Abankwa D. ColonyArea: an ImageJ plugin to automatically quantify colony formation in clonogenic assays. PloS One 2014; 9: e92444.

31. Qiu H, Durand K, Rabinovitch-Chable H, Rigaud M, Gazaille V, Clavere P et al. Gene expression of HIF-1alpha and XRCC4 measured in human samples by real-time RT-PCR using the sigmoidal curve-fitting method. BioTechniques 2007; 42: 355-362.

32. Crispi S, Calogero RA, Santini M, Mellone P, Vincenzi B, Citro G et al. Global gene expression profiling of human pleural mesotheliomas: identification of matrix metalloproteinase 14 (MMP-14) as potential tumour target. PLoS One 2009; 4: e7016.

Attribution 4.0 International License. The images or other third party material in this article are included in the article's Creative Commons license, unless indicated otherwise in the credit line; if the material is not included under the Creative Commons license, users will need to obtain permission from the license holder to reproduce the material. To view a copy of this license, visit http:// creativecommons.org/licenses/by/4.0/ 